\title{
Barreiras na implementaçâo das diretrizes de detecção precoce dos cânceres de mama e colo do útero no Brasil
}

\section{| ${ }^{1}$ Renata Oliveira Maciel dos Santos, ${ }^{2}$ Danielle Nogueira Ramos, Arn Migowski |}

Resumo: Diretrizes clínicas podem melhorar a qualidade e a segurança da atenção à saúde, reduzindo açōes inapropriadas, entretanto, a implementação desses documentos é influenciada por diversos fatores. O objetivo deste estudo foi identificar as barreiras na implementação das diretrizes para a detecçáo precoce do câncer de mama e do colo do útero no Brasil sob a perspectiva dos gestores do SUS. Trata-se de uma pesquisa transversal e exploratória com desenho quantitativo-qualitativo, com 60 gestores. As principais barreiras para o câncer de mama foram: conflitos com as sociedades de especialidades médicas (31,0\%); baixa adesão dos profissionais (20,7\%); desorganizaçấo dos serviços (17,2\%); e para o câncer do colo do útero: pouca tradição organizacional no uso de diretrizes (25,0\%); baixa adesão dos profissionais $(21,4 \%)$; desorganização dos serviços de saúde (21,4\%). Demais barreiras identificadas foram a precariedade do sistema de informação e alta rotatividade dos profissionais. Diferenças regionais se destacam na demanda inapropriada da população na Região Sul e na escassez de recursos financeiros na Regiáo Norte. Os resultados reiteram pressupostos de como a comunicação ineficiente pode impactar na prática e adesão dos profissionais e usuários às recomendaçôes e que as barreiras se relacionam a condiçóes interdependentes e de forma sinérgica se potencializam nas dimensôes práticas, sistêmicas e organizacionais.

> Palavras-chave: detecção precoce de câncer; fidelidade a diretrizes; neoplasias de mama; neoplasias de colo de útero.

\author{
1 Instituto Nacional de Câncer. \\ Rio de Janeiro-RJ, Brasil (renata. \\ santos@inca.gov.br). \\ ORCID: 0000-0002-6747-0184. \\ ${ }^{2}$ Instituto Nacional de Câncer. \\ Rio de Janeiro-RJ, Brasil \\ (daninogueira7@yahoo.com.br). \\ ORCID: 0000-0002-5876-1158. \\ ${ }^{3}$ Instituto Nacional de Câncer \\ Rio de Janeiro-RJ, Brasil (arn. \\ santos@inca.gov.br) \\ ORCID: 0000-0002-4861-2319.
}

Recebido em: 28/11/2018 Revisado em: 17/05/2019 Aprovado em: 15/08/2019 


\section{Introdução}

Diretrizes clínicas são documentos que apresentam recomendaçóes desenvolvidas com base na literatura científica e têm por intuito otimizar a atenção à saúde por meio da padronização de condutas (INSTITUTE OF MEDICINE, 2011). Idealmente, devem considerar a avaliação dos riscos, benefícios, a relação custo-benefício existente, indicando a intervenção mais adequada a cada contexto. A utilização de diretrizes ou protocolos clínicos, baseados em evidências, tem por objetivo melhorar a qualidade e a segurança da atenção à saúde, reduzindo o número de decisôes inapropriadas (MIGOWSKI; FERNANDES, 2016).

No Brasil, o Instituto Nacional de Câncer (INCA), órgão do Ministério da Saúde, é responsável pela publicação de diretrizes clínicas para a detecção precoce do câncer de mama e do colo do útero. Esses cânceres são, respectivamente, o primeiro e terceiro mais incidentes nas mulheres brasileiras, sem considerar o câncer de pele não melanoma. Essas diretrizes recomendam o rastreamento mamográfico com frequência bienal em mulheres de 50 a 69 anos e o exame citopatológico do colo do útero em mulheres de 25 a 64 anos, com periodicidade trienal após dois exames normais anuais consecutivos (MIGOWSKI et al., 2018a; BRASIL, 2015).

Apesar de recomendaçóes semelhantes a estas estarem vigentes desde 1988 para o rastreamento do câncer do colo do útero e desde 2004 para o câncer de mama, é possível observar que a prática difere de maneira considerável das diretrizes nacionais. Em 2013, segundo os dados de monitoramento nacional apenas $11 \%$ e $78 \%$ dos exames citopatológicos e $32 \%$ e $53 \%$ das mamografias de rastreamento solicitadas no Brasil seguem, respectivamente, a periodicidade e a faixa etária recomendadas pelas diretrizes (BRASIL, 2015; TOMAZELLI et al., 2017).

Apenas a publicação das diretrizes é insuficiente para provocar adesão dos profissionais de saúde e a mudança de comportamento na clínica. Para tal, estudos apontam a necessidade de planejar e executar açôes de disseminação, identificar as barreiras relacionadas aos diversos atores e direcionar estratégias para efetiva implementação dessas recomendações (FISCHER et al., 2016).

$\mathrm{Na}$ literatura internacional, as principais barreiras encontradas estáo relacionadas a fatores políticos e sociais; crença e hábitos dos profissionais; sistemas de pagamento; preferências dos usuários e características das diretrizes, como o processo de desenvolvimento e aplicabilidade. No cenário nacional, porém, esses dados são mais 
difíceis de encontrar e quando disponíveis, retratam o conhecimento e a prática dos profissionais de saúde diante dessas recomendaçôes e poucos relatam a visão dos outros atores envolvidos, especialmente dos gestores (CARLSEN; BRINGEDAL, 2011; STORMO; DE MOURA; SARAIYA, 2014; LEE et al., 2015; MORAES et al., 2016; AMARAL; GONÇALVES; SILVEIRA, 2017).

Buscando explorar esse cenário e entender os principais problemas encontrados pela gestão, este estudo teve por objetivo identificar as barreiras na implementação das diretrizes para a detecção precoce do câncer de mama e do colo do útero no Brasil, sob a perspectiva dos gestores do Sistema Único de Saúde (SUS).

\section{Metodologia}

Trata-se de uma pesquisa transversal e exploratória com desenho metodológico misto quantitativo-qualitativo do tipo complementar (DOYLE; BRADY; BYRNE, 2016). A escolha desta metodologia ocorreu para que fosse possível obter dados complementares sobre o mesmo tópico, de modo a melhor compreender as barreiras na implementação das diretrizes nacionais para a detecção precoce do câncer de mama e do colo do útero.

A coleta de dados foi realizada por meio de questionário autopreenchível, aplicado na "Oficina Nacional sobre a Implementação das Diretrizes de Detecção Precoce do Câncer”, que aconteceu, em outubro de 2016, em uma instituição pública federal na cidade do Rio de Janeiro. O evento tinha como objetivo discutir as estratégias de intervenção para a efetiva implementação das diretrizes para detecção precoce do câncer no Brasil. Para a oficina, foram convidados quatro representantes federais da área de câncer e saúde da mulher; dois representantes de cada Unidade Federativa (UF) e um representante de cada capital de UF. Os convites foram direcionados ao e-mail institucional das secretarias de saúde, sendo indicados representantes que exerciam atividades de coordenação na área tema da oficina. Cabe destacar que o Brasil é dividido em cinco regiōes e possui 27 Unidades Federativas distribuídas nas regiōes: Norte (7), Nordeste (9), Centro-Oeste (4), Sudeste (4), Sul (3); cada UF possui uma capital. As regiōes apresentam disparidades em nível socioeconômico, acesso aos serviços de saúde, entre outros (IBGE, 2018).

Ao início do evento, os gestores foram convidados a participar da pesquisa e apresentados os objetivos e o caráter voluntário da mesma, sendo entregue o questionário após a assinatura do Termo de Consentimento Livre e Esclarecido. 
O questionário era composto de uma listagem de barreiras, produzidas a partir de busca prévia na literatura científica. $\mathrm{O}$ preenchimento foi por ranqueamento em escala de 1 a 6 , sendo rotulada como "1" a barreira mais influente e " 6 " a menos influente para a implementação das diretrizes. Foi solicitado que os gestores classificassem as barreiras a partir de sua percepção de prioridade de influência na sua prática. Ao lado de cada barreira havia um espaço destinado para comentários a ser preenchido de forma livre pelos participantes e um campo aberto para o preenchimento de outras barreiras percebidas e que não estavam listadas. A última parte era composta de perguntas relacionadas ao perfil dos respondentes.

Para análise dos dados quantitativos foi realizada frequência simples para identificar a barreira mais citada e a média ponderada para obter o ranqueamento das mesmas para o Brasil e regiōes, sendo calculado o desvio-padrão de cada variável. As barreiras foram apresentadas em tabelas, representando o ranqueamento nacional e a sua posição para cada região do Brasil. As médias aritméticas das diversas escalas individuais foram agrupadas de acordo com a região do gestor. Para comparação das médias de cada barreira entre as cinco regiôes do país, foi realizada a Análise de Variância (ANOVA), sendo os pressupostos de normalidade e de homogeneidade de variâncias avaliados, respectivamente, pelo teste de Shapiro-Wilk e pelo teste de Levene. Para os casos nos quais o pressuposto de normalidade não foi atendido, foi realizado o teste não paramétrico de Kruskal-Wallis em vez de ANOVA. As análises das diferenças regionais da classificação de cada barreira foram estratificadas por tipo de câncer (colo do útero ou mama). Para ambos os testes, as diferenças entre estados foram consideradas estatisticamente significativas quando o valor de $\mathrm{p}$ foi inferior a 0,05. Todas essas análises foram feitas no programa $\mathrm{R}$ versão 3.6.0.

Para análise qualitativa, optou-se pela a de conteúdo (MINAYO, 2002), em que se pretendeu captar os sentidos, consolidando os dados a partir da perspectiva dos sujeitos, seguindo as etapas de pré-análise, exploração do material e tratamento dos resultados. Os dados foram examinados por dois pesquisadores, seguindose de leitura exaustiva, agrupamento em unidades de análise e categorização das barreiras inseridas pelos participantes. As barreiras foram apresentadas de forma descritiva. Os comentários foram analisados por dois pesquisadores, seguindo as categorias e justificativas relacionadas à influência de cada barreira e como ela é percebida pelos participantes. 
Para proteger o sigilo dos respondentes, optou-se por identificar os gestores por região e os comentários por número de participantes. O protocolo de estudo foi aprovado pelo Comitê de Ética em Pesquisa da instituição pesquisadora (CAAE: 59350416.7.0000.5274).

\section{Resultados}

Dos 85 gestores convidados para participar do evento, 64 (75,3\%) compareceram. Todos foram convidados a participar da pesquisa, sendo alcançados 60 (93,8\%) questionários respondidos. Desses 60 , seis foram eliminados devido a erros de preenchimento.

Dentre os gestores que não participaram do estudo, seja por recusa ou devido ao erro de preenchimento do questionário; quatro eram da Região Nordeste, dois do Norte, dois do Sudeste, um do Centro-Oeste e um do Sul. Apenas um estado da Região Nordeste não teve representante. Sendo assim, considerou-se que as perdas não afetaram a representatividade das regiōes.

Dos 54 questionários elegíveis, a maioria era de gestores atuantes nas regióes Norte e Nordeste, devido ao maior número de UF nessas regiōes. A maior parte dos gestores respondeu ter mais de três anos de experiência em gestáo e no cargo atual (tabela 1).

Tabela 1. Características da população do estudo, Rio de Janeiro, Brasil, 2018

\begin{tabular}{l|c|c}
\hline VARIÁVEIS & $\mathbf{N}$ & $\mathbf{\%}$ \\
\hline Sexo & & \\
\hline Feminino & $\mathbf{5 1}$ & $\mathbf{9 4 , 4}$ \\
\hline Masculino & 3 & 5,6 \\
\hline Idade & & \\
\hline$<30$ anos & 3 & 5,6 \\
\hline 30 a 49 anos & 18 & 51,9 \\
\hline 50 a 59 anos & 20 & 37 \\
\hline$\geq 60$ anos & 3 & 5,6 \\
\hline & & continua...
\end{tabular}




\begin{tabular}{|c|c|c|}
\hline VARIÁVEIS & $\mathbf{N}$ & $\%$ \\
\hline \multicolumn{3}{|l|}{ Secretaria } \\
\hline Municipal (Capital da UF) & 12 & 22,2 \\
\hline Estadual & 37 & 68,5 \\
\hline Federal & 3 & 5,6 \\
\hline Sem informação & 2 & 3,7 \\
\hline \multicolumn{3}{|l|}{ Região } \\
\hline Norte & 16 & 29,6 \\
\hline Nordeste & 17 & 31,5 \\
\hline Centro-Oeste & 9 & 16,7 \\
\hline Sudeste & 6 & 11,1 \\
\hline Sul & 6 & 11,1 \\
\hline \multicolumn{3}{|l|}{ Escolaridade } \\
\hline Doutorado & 1 & 1,9 \\
\hline Mestrado & 16 & 29,6 \\
\hline Especialização & 34 & 63 \\
\hline Graduação & 2 & 3,7 \\
\hline Ensino médio & 1 & 1,9 \\
\hline \multicolumn{3}{|l|}{ Formação educacional } \\
\hline Medicina & 10 & 18,5 \\
\hline Enfermagem & 33 & 61,1 \\
\hline Outra* & 10 & 18,5 \\
\hline Sem informação & 1 & 1,9 \\
\hline \multicolumn{3}{|l|}{ Área de atuação na secretaria } \\
\hline Câncer & 16 & 29,6 \\
\hline Saúde da Mulher & 23 & 42,5 \\
\hline Câncer e Saúde da Mulher & 5 & 9,2 \\
\hline Outra** & 6 & 11,1 \\
\hline Sem informação & 2 & 7,4 \\
\hline
\end{tabular}




\begin{tabular}{l|c|c}
\hline VARIÁVEIS & $\mathbf{N}$ & \% \\
\hline Anos em seu cargo atual & 4 & 7,4 \\
\hline$<1$ ano & 15 & 27,8 \\
\hline$\geq 1$ ano e $<3$ anos & 15 & 27,8 \\
\hline$\geq 3$ e $<5$ anos & 8 & 14,8 \\
\hline$\geq 5$ e $<8$ anos & 12 & 22,2 \\
\hline$\geq 8$ anos & & \\
\hline Anos trabalhados na gestáo & 2 & 3,7 \\
\hline$<1$ ano & 7 & 13 \\
\hline$\geq 1$ ano e $<3$ anos & 10 & 18,5 \\
\hline$\geq 3$ e $<5$ anos & 6 & 11,1 \\
\hline$\geq 5$ e $<8$ anos & 28 & 51,9 \\
\hline$\geq 8$ e $<14$ anos & 1 & 1,8 \\
\hline Sem informação & & \\
\hline Fon & éboraça propria & \\
\hline
\end{tabular}

Fonte: elaboração própria.

Os gestores foram questionados quanto ao conhecimento das diretrizes de rastreamento do INCA para o câncer de mama e do colo do útero. Para o câncer de mama, $80 \%$ das respostas em relação à faixa etária e 82\% em relação à periodicidade foram concordantes com as diretrizes do INCA/MS. Para o câncer do colo do útero esse percentual foi de $70 \%$ para a faixa etária e $56 \%$ para a periodicidade.

Em relação às barreiras listadas no questionário para a detecçáo precoce do câncer de mama, 18 (31,0\%) respondentes identificaram o conflito com as sociedades de especialidades médicas como a mais frequente, seguido de 12 (20,7\%), que apontaram a baixa adesão dos profissionais e 10 (17,2\%), a desorganização dos serviços. Para o câncer do colo do útero, 14 (25,0\%) indicaram a pouca tradição organizacional no uso de diretrizes, $12(21,4 \%)$, a baixa adesão dos profissionais e 12 (21,4\%), a desorganização dos serviços de saúde.

Quanto ao ranqueamento, a baixa adesão dos profissionais e a desorganização dos serviços ficaram como primeiro e segundo lugar, respectivamente, para os dois tipos de cânceres. O conflito com as sociedades médicas ficou em terceiro para o 
câncer de mama, sendo a menos importante para o câncer do colo do útero. Entre as regióes, destacam-se diferenças na Região Sul, com o terceiro lugar para a demanda inapropriada da população para o câncer de mama e segundo para o colo do útero, e na Regiáo Sudeste, que aponta o conflito com as sociedades médicas como terceira mais influente para o câncer do colo do útero, sendo menos influente que a organização dos serviços. Apenas para a barreira "Desorganização dos serviços" para câncer do colo do útero a diferença entre regiôes foi estatisticamente significativa (tabelas 2 e 3).

Tabela 2. Ranqueamento por média e desvio-padrão das barreiras para a implementação das diretrizes de detecção precoce do câncer de mama, Brasil e regióes, Rio de Janeiro, Brasil, 2018

\begin{tabular}{|c|c|c|c|c|c|c|c|}
\hline Barreira & Brasil & Norte & Nordeste & Centro-Oeste & Sul & Sudeste & p valor \\
\hline \multirow{2}{*}{$\begin{array}{l}\text { Baixa adesão dos } \\
\text { profissionais }\end{array}$} & $1^{\circ}(2,57)$ & $1^{\circ}(2,31)$ & $1^{\circ}(2,47)$ & $3^{\circ}(3,30)$ & $2^{\circ}(3,0)$ & $1^{\circ}(2,33)$ & \multirow{2}{*}{$\begin{array}{c}\text { valor-p* } \\
0,157\end{array}$} \\
\hline & DP: 1,4 & DP: 1,6 & DP: 1,5 & DP: 1,1 & DP: 1,0 & DP: 0,8 & \\
\hline \multirow{2}{*}{$\begin{array}{l}\text { Desorganização } \\
\text { dos serviços }\end{array}$} & $2^{\circ}(3,20)$ & $3^{\circ}(3,50)$ & $3^{\circ}(3,47)$ & $1^{\circ}(2,0)$ & $1^{\circ}(2,50)$ & $5^{\circ}(4,0)$ & \multirow{2}{*}{$\begin{array}{c}\text { valor-p* }^{*} \\
0,052\end{array}$} \\
\hline & DP: 1,6 & DP: 1,2 & DP: 2,0 & DP: 1,0 & DP:0,5 & DP: 1,9 & \\
\hline \multirow{2}{*}{$\begin{array}{l}\text { Conflito com } \\
\text { as sociedades } \\
\text { médicas }\end{array}$} & $3^{\circ}(3,29)$ & $4^{\circ}(3,68)$ & $2^{\circ}(3,0)$ & $2^{\circ}(3,0)$ & $4^{\mathrm{o}}(3,33)$ & $2^{\circ}(2,83)$ & \multirow{2}{*}{$\begin{array}{c}\text { valor-p* } \\
0,874\end{array}$} \\
\hline & $\mathrm{DP}: 2,0$ & DP:2,0 & DP:2,0 & DP: 2,3 & $\mathrm{DP}: 2,5$ & DP: 1,1 & \\
\hline \multirow{2}{*}{$\begin{array}{l}\text { Pouca tradição } \\
\text { organizacional no } \\
\text { uso das diretrizes }\end{array}$} & $4^{\circ}(3,88)$ & $5^{\circ}(3,87)$ & $5^{\circ}(3,70)$ & $5^{\circ}(4,34)$ & $5^{\circ}(4,50)$ & $3^{\circ}(3,0)$ & \multirow{2}{*}{$\begin{array}{c}\text { valor-p** } \\
0,435\end{array}$} \\
\hline & DP: 1,5 & DP: 1,4 & DP:1,6 & DP:2,1 & DP: 1,5 & DP: 1,7 & \\
\hline \multirow{2}{*}{$\begin{array}{l}\text { Demanda } \\
\text { inapropriada da } \\
\text { população }\end{array}$} & $5^{\circ}(4,01)$ & $6^{\circ}(4,12)$ & $4^{\circ}(3,52)$ & $6^{\circ}(5,11)$ & $3^{\circ}(3,16)$ & $4^{\circ}(3,83)$ & \multirow{2}{*}{$\begin{array}{c}\text { valor-p* } \\
0,189\end{array}$} \\
\hline & DP: 1,6 & DP: 1,8 & DP:1,3 & DP: 0,8 & DP: 2,4 & DP: 1,8 & \\
\hline \multirow{2}{*}{$\begin{array}{l}\text { Recursos } \\
\text { financeiros } \\
\text { escassos }\end{array}$} & $6^{\circ}(4,01)$ & $2^{\circ}(3,43)$ & $6^{\circ}(4,29)$ & $4^{\circ}(3,66)$ & $6^{\circ}(4,50)$ & $6^{\circ}(5,33)$ & \multirow{2}{*}{$\begin{array}{c}\text { valor-p** } \\
0,108\end{array}$} \\
\hline & DP: 1,5 & DP: 1,4 & DP: 1,7 & DP: 1,4 & DP: 0,8 & DP: 1,7 & \\
\hline
\end{tabular}

p valor* teste Kruskal-Wallis

p valor ${ }^{* *}$ teste ANOVA

$\mathrm{O}$ valor de p refere-se à comparação entre regióes do país para cada barreira.

Fonte: elaboração própria. 
Tabela 3. Ranqueamento por média e desvio-padrão das barreiras relacionadas à implantação das diretrizes de deteç̧áo precoce do câncer do colo do útero, Brasil e regiôes, Rio de Janeiro, Brasil, 2018

\begin{tabular}{|c|c|c|c|c|c|c|c|}
\hline $\begin{array}{l}\text { Principal } \\
\text { barreira }\end{array}$ & Brasil & Norte & Nordeste & Centro-Oeste & Sul & Sudeste & p valor \\
\hline \multirow{2}{*}{$\begin{array}{l}\text { Baixa adesão dos } \\
\text { profissionais }\end{array}$} & $1^{\circ}(2,48)$ & $1^{\circ}(2,62)$ & $1^{\circ}(2,23)$ & $3^{\circ}(3,25)$ & $3^{\circ}(3,00)$ & $1^{\circ}(2,83)$ & \multirow{2}{*}{$\begin{array}{c}\text { valor-p* } \\
0,241\end{array}$} \\
\hline & DP: 1,2 & DP: 1,4 & DP:1,2 & DP: 1,0 & DP:1,2 & DP: 1,2 & \\
\hline \multirow{2}{*}{$\begin{array}{l}\text { Desorganização } \\
\text { dos serviços }\end{array}$} & $2^{\circ}(3,22)$ & $4^{\circ}(3,75)$ & $4^{\circ}(3,41)$ & $1^{\circ}(2,12)$ & $1^{\circ}(2,16)$ & $5^{\circ}(4,50)$ & \multirow{2}{*}{$\begin{array}{c}\text { valor-p ** } \\
0,037\end{array}$} \\
\hline & DP:1,7 & DP: 1,7 & DP:1,6 & DP: 1,6 & DP:1,3 & DP: 1,8 & \\
\hline \multirow{2}{*}{$\begin{array}{l}\text { Pouca tradição } \\
\text { organizacional no } \\
\text { uso das diretrizes }\end{array}$} & $3^{\circ}(3,27)$ & $2^{\circ}(3,06)$ & $2^{\circ}(3,23)$ & $2^{\circ}(2,33)$ & $4^{\circ}(4,50)$ & $2^{\circ}(3,0)$ & \multirow{2}{*}{$\begin{array}{c}\text { valor-p* } \\
0,319\end{array}$} \\
\hline & DP: 1,7 & DP: 1,8 & DP: 1,5 & DP: 1,4 & DP: 1,8 & DP: 1,7 & \\
\hline \multirow{2}{*}{$\begin{array}{l}\text { Demanda } \\
\text { inapropriada da } \\
\text { população }\end{array}$} & $4^{\circ}(3,94)$ & $6^{\circ}(4,31)$ & $3^{\circ}(3,29)$ & $5^{\circ}(4,30)$ & $2^{\circ}(2,83)$ & $4^{\circ}(3,83)$ & \multirow{2}{*}{$\begin{array}{c}\text { valor-p* } \\
0,249\end{array}$} \\
\hline & DP: 1,6 & DP: 1,5 & DP: 1,4 & DP:0,9 & DP: 2,1 & DP 1,2 & \\
\hline \multirow{2}{*}{$\begin{array}{l}\text { Recursos } \\
\text { financeiros } \\
\text { escassos }\end{array}$} & $5^{\circ}(4,11)$ & $5^{\circ}(3,93)$ & $5^{\circ}(4,05)$ & $4^{\circ}(3,37)$ & $5^{\circ}(4,83)$ & $6^{\circ}(4,83)$ & \multirow{2}{*}{$\begin{array}{c}\text { valor-p* } \\
0,294\end{array}$} \\
\hline & DP: 1,6 & DP: 1,5 & DP:1,6 & DP:1,9 & DP: 1,1 & DP: 1,4 & \\
\hline \multirow{2}{*}{$\begin{array}{l}\text { Conflito com } \\
\text { as sociedades } \\
\text { médicas }\end{array}$} & $6^{\circ}(4,40)$ & $3^{\circ}(3,62)$ & $6^{\circ}(4,70)$ & $6^{\circ}(5,44)$ & $6^{\circ}(5,00)$ & $3^{\circ}(3,16)$ & \multirow{2}{*}{$\begin{array}{c}\text { valor-p** } \\
0,066\end{array}$} \\
\hline & DP: 1,8 & DP: 2,0 & DP: 1,7 & DP: 0,5 & DP: 2,0 & DP: 2,2 & \\
\hline
\end{tabular}

p valor* teste Kruskal-Wallis

p valor ${ }^{* *}$ teste ANOVA

$\mathrm{O}$ valor de p refere-se à comparação entre regiōes do país para cada barreira.

Fonte: elaboração própria.

Dos 54 participantes do estudo, 26 (48\%) fizeram alguma contribuição escrita, sendo $17(31,4 \%)$ por comentário, e $11(20,0 \%)$ identificaram outras barreiras, além das listadas no questionário (quadro 1). 
Quadro 1. Outras barreiras* identificadas para a implementação das Diretrizes de Detecçấo Precoce dos cânceres de mama e do colo do útero, Rio de Janeiro, Brasil, 2018

\begin{tabular}{|l|c|c|}
\hline Câncer de mama & Região & N \\
\hline Alta rotatividade dos profissionais da atenção básica. & Norte/Sudeste & 4 \\
\hline $\begin{array}{l}\text { Sistema de Informação de Câncer (SISCAN) está precário e dificulta } \\
\text { o monitoramento dos casos. }\end{array}$ & Nordeste & 2 \\
\hline Pactuação de serviço com prestadores particulares. & Centro-Oeste & 1 \\
\hline $\begin{array}{l}\text { Organizaçáes náo governamentais que divulgam orientaçōes das } \\
\text { sociedades científicas e seus conflitos de interesses. }\end{array}$ & Sudeste & 1 \\
\hline Déficit de Recursos Humanos. & Sul & 1 \\
\hline Pouca participaçáo da academia [universidades]. & Norte & 1 \\
\hline Colo do útero & Norte & 3 \\
\hline Alta rotatividade de profissionais. & Nordeste & 2 \\
\hline $\begin{array}{l}\text { Sistema de Informaçáo de Câncer (SISCAN) está precário e dificulta } \\
\text { o monitoramento dos casos. }\end{array}$ & $\begin{array}{c}\text { Sul } \\
\text { Impossibilidade de coleta do citopatológico pelos auxiliares e técnicos } \\
\text { de enfermagem. }\end{array}$ & 2 \\
\hline Início da idade sexual precoce das mulheres. & Sul & 1 \\
\hline Medo, vergonha ou desconhecimento [das mulheres] sobre o exame. & Sul & 1 \\
\hline
\end{tabular}

* barreiras que não estavam listadas no questionário.

Fonte: elaboração própria.

Os comentários evidenciaram que as barreiras identificadas se concentram no conhecimento e aceitação dos profissionais em relação ao uso das diretrizes e que a divulgação de recomendaçôes divergentes pode dificultar a implementação das mesmas, especialmente pela influência da mídia e da população frente aos profissionais, como destacado nas falas abaixo:

Há desconhecimento da diretriz (P3).

Devido às informações provenientes dos diversos órgãos, muitos profissionais não aceitam as recomendaçóes preconizadas (P6).

Os profissionais indicam o Papanicolau por "n" motivos que não rastreamento P25). 
A desorganização dos serviços de saúde interfere no planeja mento e monitoramento das açóes, assim como a falta de recursos dificulta o investimento em estratégias de disseminação, como distribuição de materiais educativos e a realização de eventos para capacitação, conforme apresentado pelos comentários:

Não organiza o serviço embasado em evidências (P44).

Municípios não conhecem a população que deve realizar o exame (P22).

A adesão às recomendaçôes é prejudicada pela pouca tradição institucional em utilizar esses documentos e de organizar sua prática a partir das evidências científicas, sendo destacada pelos gestores como uma questão institucional que reflete na adesão individual dos profissionais.

Poucos [profissionais] conhecem a evidência científica (P32).

Também foram identificados fatores relacionados à população, como a falta de informação que pode gerar demanda inadequada, podendo estar relacionada a práticas historicamente já consolidadas, que se sobrepõe à evidência científica.

Por falta de informação as mulheres são mais expostas a procurar exames (P50).

Falta de informação sobre HPV e lesóes precursoras leva a achar que o Papanicolau na adolescência seja indicado (P23).

A população acostumou a fazer Papanicolau anualmente e a partir do início da atividade sexual (P12).

\section{Discussão}

Embora a detecção precoce dos cânceres de colo do útero e de mama seja considerada açôes prioritárias de saúde no Brasil, até onde foi possível saber, esse é o primeiro estudo publicado a explorar as barreiras de implementação na visão de gestores em nível nacional. De uma forma geral, as barreiras tidas como mais influentes foram a baixa adesão dos profissionais de saúde e a desorganização dos serviços.

Mesmo com a grande parte dos gestores indicando concordância com as diretrizes do INCA/MS, a baixa adesão a essas recomendaçôes se conecta com a falta de conhecimento sobre a existência da diretriz, seu processo de elaboração, falta de concordância com as recomendaçóes e resistência quanto à mudança de comportamento, principalmente dos profissionais da prática clínica. Esses pontos podem ser minimizados com estratégias de divulgação, com foco nesses profissionais, líderes de opinião e população (COOK et al., 2018). 
A adesão dos profissionais às diretrizes é a barreira mais estudada dentro da temática da implementação de diretrizes. Em uma pesquisa realizada pelo New England Healthcare Institute, foi identificado que a maioria dos profissionais médicos se classificou como usuários leves de diretrizes, referindo pouca adesão a esses documentos (HOPE KENEFICK; JASON; FLEISHMAN, 2008).

Um estudo realizado no Brasil, com profissionais de 1.600 Unidades de Saúde da Família, mostrou que a maioria dos médicos e enfermeiros $(68,7 \%$ e 76,9\%, respectivamente) declarou que as recomendaçôes nacionais para rastreamento do câncer do colo do útero são muito influentes na sua unidade de saúde; entretanto, na prática, a maioria não segue as recomendaçóes. Noventa e três por cento dos profissionais relataram rastrear anualmente mulheres para o câncer do colo do útero e 75,1\% relataram iniciar o rastreamento em mulheres antes dos 25 anos, sendo os enfermeiros os que mais realizavam ambas as práticas (STORMO; DE MOURA; SARAIYA, 2014).

Entretanto, não é só a falta de conhecimento que explica a dificuldade de implementar as diretrizes. Outros possíveis determinantes identificados foram a desorganização dos serviços de saúde, alta rotatividade de profissionais da atenção básica, dificultando ações de capacitação, e o conflito com diretrizes de sociedades médicas. A ausência de programas organizados de rastreamento dificulta, sabidamente, a adesão às diretrizes de rastreamento, além da prática da medicina defensiva (MIGOWSKI; DIAS; NADANOVSKY, 2018).

A alta rotatividade dos profissionais de saúde foi fortemente apresentada por gestores da Região Norte, que é uma das regiôes com menor nível socioeconômico de desenvolvimento e possui dificuldades na fixação de profissionais (IBGE, 2018). A literatura destaca que em locais com menor estrutura financeira e condiçóes precárias de trabalho, a rotatividade e a falta de recursos humanos são alguns dos principais entraves para a organização dos serviços de saúde (BORRELLI, 2004). Os principais fatores desencadeantes se relacionam com a falta de motivação, vínculos empregatícios precários, remuneração insatisfatória, dificuldades de relacionamento político e interpessoal e falta de políticas de fixação de profissionais (MEDEIROS et al., 2010).

O conflito com as sociedades médicas se dá, essencialmente, devido à divergência nas recomendaçôes de faixa etária e periodicidade do rastreamento. Para o câncer de mama, o rastreamento mamográfico é preconizado por essas instituiçôes na idade 
de 40 anos e periodicidade anual, sendo diferente da recomendação do INCA/ MS, que é a partir dos 50 anos e bienal (MIGOWSKI et al., 2018b). Embora essas últimas possuam maior rigor metodológico em seu processo de elaboração, as recomendações de sociedades médicas têm, frequentemente, maior difusão entre a população em geral, seja por maior acesso aos veículos de comunicação de massa e apoio de organizaçôes não governamentais (ONGs), e também entre os profissionais de saúde, seja por periódicos científicos próprios ou por meio de outros canais de comunicação diretos com os médicos (MIGOWSKI et al., 2018b).

Essa influência das sociedades médicas nas práticas de rastreamento de câncer também é observada em outros países. O estudo realizado pela Força Tarefa Americana demonstrou que a prática dos profissionais diferia consideravelmente de suas recomendaçóes para o rastreamento do câncer de mama e se aproximava das preconizadas pelas sociedades médicas (HINZ et al., 2011).

A demanda inapropriada da população teve maior destaque na Região Sul para os dois tipos de câncer e se relaciona com a solicitação ativa de rastreamento inadequado ou resistência da mulher em aceitar as recomendaçóes descritas nas diretrizes. Acredita-se que esse fato decorre, sobretudo, pela comunicação ineficaz ou equivocada com a população, uma vez que essa região também apontou barreiras relacionadas a fatores individuais - início da atividade sexual e desconhecimento da mulher - como impedimentos para seguir a recomendação do rastreio citológico. Tais resultados sugerem que as recomendaçôes das sociedades médicas podem ter maior difusão na população em locais com maiores condições socioeconômicas e maior participação do sistema privado de saúde, já que essa barreira foi a menos influente na Região Norte, que identificou a escassez de recursos financeiros como a mais influente (CARLSEN; BRINGEDAL, 2011; IBGE, 2016). A periodicidade e a faixa etária para a realização do rastreio citológico são constantemente relatadas na literatura como uma prática divergente das recomendadas nas diretrizes, pois açôes já enraizadas na rotina da população e dos profissionais dificilmente são desconstruídas com facilidade (VALENTE et al., 2009).

$\mathrm{O}$ risco mais comum do rastreamento em faixas etárias e periodicidades sem evidência de benefício está relacionado aos resultados incorretos, como os falsopositivos e falso-negativos. Estes podem levar à realização de outros exames, por vezes invasivos, para confirmar ou refutar o diagnóstico, gerando danos emocionais, financeiros e biológicos desnecessários. Acrescenta-se aos riscos o sobrediagnóstico e 
o sobretratamento, ou seja, diagnóstico e tratamento de cânceres que não causariam sinais, sintomas ou risco de morte. Já para o câncer do colo do útero, a realização do exame em mulheres abaixo dos 25 anos pode levar ao tratamento de lesôes precursoras provavelmente reversíveis e, assim, acarretar maior risco de morbidade obstétrica e neonatal (BRASIL, 2015; 2016).

Para o rastreamento do câncer, essa discussão é especialmente relevante, pois procedimentos e tecnologias que são, frequentemente, divulgadas como capazes de identificar precocemente alguns tipos de tumores podem expor indivíduos aparentemente saudáveis a intervençôes danosas. Além de comprometerem a sustentabilidade do sistema de saúde ao proporcionar gastos desnecessários sem trazer os benefícios esperados (STEIN; LANG; MIGOWSKI, 2018).

A pouca tradição organizacional do uso de diretrizes está relacionada a uma cultura institucional, que repercute na decisão individual do profissional. Estudos referem que a resistência em usar diretrizes pode estar relacionada ao sentimento da perda de autonomia dos profissionais, quando seguem protocolos. Já outros defendem que o uso de protocolos clínicos não considera as características individuais e que, por si só, não deveriam determinar o padrão de conduta (LUGTENBERG et al., 2011; CATTAMANCHI et al., 2015; BECKER; LOCH; REIS, 2018; COOK et al., 2018).

Para enfrentar esse problema, autores sugerem fortalecer a educação permanente do público-alvo (FISCHER et al., 2016). As instituiçôes de ensino foram apontadas como potenciais facilitadoras nesse processo, uma vez que participam da formação, capacitação e atualização dos profissionais de saúde, podendo se configurar como uma parceira na disseminação desse conteúdo. Maior integração entre pesquisadores e formuladores de políticas também é apontada como uma estratégia facilitadora, uma vez que pode aproximar o uso da evidência científica à prática clínica (OLIVER et al., 2014).

Outro estudo também realizado no Brasil (BECKER; LOCH; REIS, 2018) apresenta as barreiras organizacionais e pessoais como as principais dificuldades percebidas pelos gestores do Estado do Paraná para a tomada de decisão baseada em evidências. As organizacionais se relacionam à falta de planejamento e gestão, a características regionais e culturais da população, e as barreiras pessoais se referem à ausência de incentivo e dificuldades para trabalhar com evidências científicas e à falta de capacitação e qualificação profissional. Como recomendação desse estudo, os gestores apontam a necessidade de capacitar a equipe de trabalho, envolvendo 
profissionais administrativos e os que estão mais próximos da população, como a equipe multiprofissional e agentes de saúde (BECKER; LOCH; REIS, 2018).

O manual do National Health Service (NHS) apresenta as estratégias educacionais mais indicadas para proporcionar a mudança de comportamento nos profissionais. A publicação destaca a distribuição de materiais educativos (impressos e eletrônicos), a realização de oficinas presencias com o uso de metodologias ativas e o monitoramento das açóes dos profissionais por auditoria e feedback. O uso de lembretes e a avaliação do desempenho dos serviços através de indicadores de qualidade também são associados a bons resultados (CHAPLIN, 2008; STEIN; LANG; MIGOWSKI, 2018).

A desorganização dos serviços de saúde foi apontada em primeiro lugar nas regiôes Sul e Centro-Oeste para os dois tipos de câncer, ficando em colocaçóes de pouco destaque nas demais regióes, resultando em diferença estatisticamente significativa entre as regiōes do país. Esta barreira está associada às dificuldades na padronização de processos e procedimentos e no monitoramento e avaliação das atividades (FISCHER et al., 2016). Esta última é frequentemente citada em estudos de implementação, pois a indisponibilidade de recursos necessários para monitorar as estratégias implantadas resulta em falhas no processo e, por vezes, em insucesso (KARBACH et al., 2011; LUGTENBERG et al., 2011; CATTAMANCHI et al., 2015).

Os sistemas de informação são ferramentas fundamentais para o monitoramento, porém dificuldades no funcionamento pode causar entraves na avaliação da implementação, assim como impedir o diagnóstico situacional (BRASIL, 2013). A dificuldade de acessar as informaçóes pelo Sistema de Informação de Câncer (SISCAN) foi mencionada para os dois tipos de câncer.

O SISCAN é disponibilizado na plataforma web, integrando os sistemas de informação do câncer do colo do útero e do câncer de mama e tem como objetivo registrar a solicitação de exames e seus resultados, seguimento dos exames alterados e gerar dados que subsidiem o monitoramento e a avaliação dos casos (BRASIL, 2013). Esse sistema foi implantado visando aperfeiçoar os anteriores, SISMAMA e SISCOLO, e permitiria monitorar as açóes desenvolvidas pelos profissionais quanto ao rastreamento, diagnóstico e tratamento desses cânceres. Contudo, na ocasiáo, gestores relataram problemas que prejudicaram o monitoramento das açôes de vigilância desses cânceres, como dificuldade de acompanhamento dos indicadores locais, avaliação e sistematização das informaçôes referentes aos exames de rastreamento e diagnóstico. 
Outra barreira identificada foi a impossibilidade da coleta do citopatológico pelos técnicos de enfermagem. No SUS, a coleta está autorizada para algumas categorias de profissionais de saúde, dentre essas, o técnico de enfermagem. Segundo o INCA, essa é uma estratégia para ampliação da oferta do exame a toda população e assim melhorar os indicadores de cobertura (BRASIL, 2011). Entretanto, a resolução do Conselho Federal de Enfermagem restringe a coleta desse exame apenas para o enfermeiro, indicando como atividade privativa dessa categoria (COFEN, 2011). Os gestores, ao indicarem essa limitação, apontam para impactos ocasionados na oferta desse exame, impactando nas taxas de cobertura da população-alvo.

Como limitação do presente estudo, destaca-se o método de coleta de dados que não permitiu um tempo de entrevista individual de cada participante, o que limita a exploração de maiores detalhes sobre cada barreira apontada. Outro ponto é que o estudo retrata a percepção de um dos atores do processo, não alcançando todos os envolvidos (profissionais de saúde, usuários, formuladores de políticas). $\mathrm{O}$ fato de a maioria das diferenças regionais não ter sido estatisticamente significativas, já era esperado em virtude do pequeno número de indivíduos participantes, muito embora seja representativo da categoria escolhida para a pesquisa (gestores).

Entretanto, o foco nos gestores também é um dos pontos fortes deste artigo, uma vez que o estado da arte para esse tema concentra estudos que descrevem a percepção de profissionais de saúde e poucos descrevem a visão dos gestores. $\mathrm{O}$ estudo também analisa as barreiras a partir de uma perspectiva mais ampla, permitindo ter um panorama regional, podendo ser complementado por estudos locais no futuro. Tal análise pode auxiliar no planejamento e execução de ações que busquem aumentar a adesão às diretrizes baseadas em evidências, considerando as diferenças e especificidades apontadas.

O presente artigo também discute pressupostos de como a comunicação ineficiente das informaçóes pode impactar na prática e adesão dos profissionais e usuários às recomendaçôes nacionais e de como, por vezes, as barreiras se relacionam a múltiplas condiçôes interdependentes e, de certa forma, sinérgicas, que se potencializam umas às outras, tanto às dimensôes, pessoais, sistêmicas e organizacionais, que se expressaram no processo de implementação.

Nesta direção, projetamos a necessidade de investigar a percepção de outros atores envolvidos e de lançar mão de estratégias que visem à superação das barreiras apontadas, de modo a impactar na qualidade da atençáo prestada às mulheres. 
Destaca-se que os cânceres de mama e do colo do útero possuem diferenças nas taxas de incidência e de mortalidade dentro das regiōes do Brasil. O câncer de mama é o tipo de câncer mais frequente em todas as regiôes, exceto na Região Norte, onde o câncer do colo do útero ocupa a primeira posição. Sabido que as açôes de detecção precoce aliadas ao tratamento oportuno geram impacto nas taxas de mortalidade do câncer de mama e do colo do útero, a exemplo de diversos países que possuem recomendaçóes padronizadas para seu rastreamento, torna-se necessário investir em medidas que busquem aumentar a adesão a essas recomendaçôes, considerando o cenário local, com estratégias educacionais focadas na mudança de comportamento, maior difusão das diretrizes, além de esforços para a organização dos serviços, garantindo acesso, seguimento e tratamento dos casos. ${ }^{1}$

\section{Agradecimentos}

A Caroline Madalena, pelas sugestôes sobre a apresentação dos resultados nas tabelas, e a Nathan Mendes, pelas sugestôes na concepção do instrumento de coleta de dados.

\section{Referências}

AMARAL, M. S.; GONÇALVES, A. G.; SILVEIRA, L. C. G. Prevenção do câncer de colo de útero: a atuação do profissional enfermeiro nas unidades básicas de saúde. Revista Científica FacMais, v. VIII, n. 1, fev./mar., 2017.

BECKER, L. A.; LOCH, M. R.; REIS, R. S. Barreiras percebidas por diretores de saúde para tomada de decisão baseada em evidências. Revista Panamericana de Salud Pública, v. 41, p. e147, 3 maio 2018.

BORRELLI, F. R. G. A rotatividade dos profissionais de saúde na zona rural de Sergipe: um problema a ser enfrentado. Rio de Janeiro: Escola Nacional de Saúde Pública/ Departamento de Planejamento e Gestão, 2004.

CARLSEN, B.; BRINGEDAL, B. Attitudes to clinical guidelines - do GPs differ from other medical doctors? BMJ Quality \& Safety, v. 20, n. 2, p. 158-162, 1 fev. 2011.

CATTAMANCHI, A. et al. Health worker perspectives on barriers to delivery of routine tuberculosis diagnostic evaluation services in Uganda: a qualitative study to guide clinic-based interventions. BMC Health Services Research, v. 15, 22 jan. 2015.

CHAPLIN, S. How to change practice - a NICE guide to overcoming the barriers. Prescriber, v. 19, n. 2, p. 47-50, 19 jan. 2008. 
CONSELHO FEDERAL DE ENFERMAGEM. Resolução COFEN No 381/2011. BrasíliaDF, 2011.

COOK, D. A. et al. Practice variation and practice guidelines: Attitudes of generalist and specialist physicians, nurse practitioners, and physician assistants. Plos One, v. 13, n. 1, p. e0191943, 31 jan. 2018.

DOYLE, L.; BRADY, A.-M.; BYRNE, G. An overview of mixed methods research. Journal of Research in Nursing, p. 11, 2016.

FISCHER, F. et al. Barriers and Strategies in Guideline Implementation-A Scoping Review. Healthcare (Basel, Switzerland), v. 4, n. 3, 29 jun. 2016.

HINZ, E. K. et al. Physician knowledge of and adherence to the revised breast cancer screening guidelines by the United States Preventive Services Task Force. American Journal of Obstetrics \& Gynecology, v. 205, n. 3, p. 201.e1-201.e5, 1 set. 2011.

HOPE KENEFICK; JASON, L.; FLEISHMAN, V. Improving Physician Adherence to Clinical Practice Guidelines. Barriers and Strategies for Change. New England Healthcare Institute, fev. 2008.

INSTITUTO BRASILEIRO DE GEOGRAFIA E ESTATÍSTICA. Sintese de indicadores sociais: uma análise das condiçôes de vida da população brasileira. Coordenação de População e Indicadores Sociais. Rio de Janeiro: IBGE, 2018 Disponível em: < https://biblioteca.ibge.gov. br/visualizacao/livros/liv101629.pdf >.

BRASIL. Ministério da Saúde. Instituto Nacional de Câncer. Diretrizes Brasileiras para o rastreamento do Câncer do Colo do Útero. Rio de Janeiro, 2016.

. Diretrizes para a deteç̧áo precoce do câncer de mama no Brasil. Rio de Janeiro: INCA, 2015. . Sistema de Informação do Câncer Manual preliminar para apoio à implantação, 2013. Disponível em: <file://C:/Users/2248041/Downloads/Manual\%20preliminar\%20SISCAN.pdf>.

. Ofício n III/i2011 Gab. INCA, 2011. Disponível em: <http://www2.inca.gov.br/wps/ wcm/connect/8d6cd1804eb684a18af99af11fae00ee/Of\%C3\%ADcio+n\%C2\%BA+717.2011Gab.INCA\%2C+de+30.08.11.pdf?MOD=AJPERES\&CACHEID=8d6cd1804eb684a18af99a f11fae00ee>. Acesso em: 24 ago. 2018.

INSTITUTE OF MEDICINE(US) COMMITTEE ON STANDARDS FORDEVELOPING TRUSTWORTHY CLINICAL PRACTICE GUIDELINES. Clinical Practice Guidelines We Can Trust. Washington (DC): National Academies Press (US), 2011.

KARBACH, U. et al. Physicians' knowledge of and compliance with guidelines: an exploratory study in cardiovascular diseases. Deutsches Arzteblatt International, v. 108, n. 5, p. 61-69, fev. 2011. 
LEE, P. Y. et al. Healthcare professionals' and policy makers' views on implementing a clinical practice guideline of hypertension management: a qualitative study. PloS One, v. 10, n. 5, p. e0126191, 2015.

LUGTENBERG, M. et al. Perceived barriers to guideline adherence: a survey among general practitioners. BMC family practice, v. 12, p. 98, 22 set. 2011.

MEDEIROS, C. R. G. et al. A rotatividade de enfermeiros e médicos: um impasse na implementação da Estratégia de Saúde da Família. Ciência \& Saúde Coletiva, v. 15, n. suppl 1, p. 1521-1531, jun. 2010.

MIGOWSKI, A. et al. Diretrizes para detecção precoce do câncer de mama no Brasil. II Novas recomendaçóes nacionais, principais evidências e controvérsias. Cadernos de Saúde Pública, v. 34, n. 6, 2018a.

- Diretrizes para detecção precoce do câncer de mama no Brasil. I - Métodos de elaboração. Cadernos de Saúde Pública, v. 34, n. 6, 21 jun. 2018b.

MIGOWSKI, A.; DIAS, M. B. K.; NADANOVSKY, P. Diretrizes para detecção precoce do câncer de mama no Brasil. III - Desafios à implementação. Cad. Saúde Pública, p. 14, 2018.

MIGOWSKI, A.; FERNANDES, M. M. Diretrizes metodológicas/ Elaboração de diretrizes clinicas. Brasília: Ministério da saúde, 2016.

MINAYO, M. C. DE S. Pesquisa social. Teoria, método e criatividade. 28. ed. [s.1.]: Vozes, 2002.

MORAES, D. C. DE et al. Opportunistic screening actions for breast cancer performed by nurses working in primary health care. Revista da Escola de Enfermagem da USP, v. 50, n. 1, p. 14-21, fev. 2016.

OLIVER, K. et al. A systematic review of barriers to and facilitators of the use of evidence by policymakers. BMC health services research, v. 14, p. 2, 3 jan. 2014.

STEIN, A.; LANG, E.; MIGOWSKI, A. Implementing clinical guidelines: a need to follow recommendations based on the best evidence available. Rev Bras Epidemiol, 2018.

STORMO, A. R.; DE MOURA, L.; SARAIYA, M. Cervical cancer-related knowledge, attitudes, and practices of health professionals working in Brazil's network of primary care units. The Oncologist, v. 19, n. 4, p. 375-382, abr. 2014.

TOMAZELLI, J. G. et al. Assessment of actions for breast cancer early detection in Brazil using process indicators: a descriptive study with Sismama data, 2010-2011. Epidemiologia e Serviços de Saúde, v. 26, n. 1, p. 61-70, mar. 2017.

VALENTE, C. A. et al. Conhecimento de mulheres sobre o exame de Papanicolau. Revista da Escola de Enfermagem da USP, v. 43, n. esp. 2, p. 1193-1198, dez. 2009. 


\section{Nota}

${ }^{1}$ R. O. M. dos Santos idealizou o projeto, realizou a pesquisa, coleta, análise e discussão dos dados, redigiu e revisou o artigo. D. N. Ramos participou da elaboração da pesquisa, análise dos dados e revisão do manuscrito. A. Migowski participou da realização das análises estatísticas, elaboraçáo do instrumento de coleta e revisão do texto. 


\section{Abstract}

\section{Barriers to implementing early breast and cervical cancer detection guidelines in Brazil}

Clinical guidelines can improve the quality and safety of health care by reducing inappropriate actions. However, the implementation of these documents is influenced by several factors. This study aimed to identify the barriers in the implementation of guidelines for early breast and cervical cancer detection in Brazil, as seen from the public health managers (SUS) perspective. This is a cross-sectional and exploratory research with quantitative-qualitative design, involving 60 managers. The implementation main barriers for breast cancer were: medical society conflicts (31.0\%), low professionals' adherence (20.7\%); and disorganization of health services (17.2\%). For the cervical cancer, these are: little organizational tradition in the use of guidelines (25.0\%); low professionals' adherence (21.4\%); and disorganization of health services (21.4\%). Other identified barriers were the precariousness of the information health system and high turnover of professionals. Regional differences stood out in the inappropriate demand of the population in the South Region and lack of financial resources in the North Region. The results reinforce the fact that inefficient communication does affect the medical practice.

Moreover, the adherence of both professionals and users to recommendations are related to interdependent and synergistic conditions that become more relevant at the practical, systemic, and organizational dimensions.

> Keywords: early detection of cancer; guideline adherence; breast neoplasms; cervical cancer. 